\title{
Light-Induced Translation of
}

\section{Motorized Molecules on a Surface}

\begin{abstract}
Alex Saywell ${ }^{1,2}$, Anne Bakker', Johannes Mielke ${ }^{l \#}$, Takashi Kumagai ${ }^{1}$, Martin Wolf ${ }^{1}$, Victor Garcia-López ${ }^{3}$, Pinn-Tsong Chiang ${ }^{3}$, James M. Tour ${ }^{3 *}$, and Leonhard Grill1, ${ }^{4 *}$
\end{abstract}

${ }^{1}$ Department of Physical Chemistry, Fritz-Haber-Institute of the Max-Planck Society, 14195 Berlin, Germany.

${ }^{2}$ School of Physics \& Astronomy, The University of Nottingham, Nottingham, NG7 2RD, United Kingdom.

${ }^{3}$ Departments of Chemistry and Materials Science and NanoEngineering, and the Smalley Institute for Nanoscale Science and Technology, Rice University, Houston, Texas 77005, United States.

${ }^{4}$ Department of Physical Chemistry, University of Graz, Heinrichstrasse 28, 8010 Graz, Austria.

\# Present address: BAM Bundesanstalt für Materialforschung und -prüfung, Berlin, Germany.

*Corresponding authors J. M. T. (tour@rice.edu) or L. G. (leonhard.grill@uni-graz.at).

KEYWORDS - Diffusion, Molecular devices, Molecular motor, Photoexcitation, Photochemistry, Scanning probe microscopy 


\begin{abstract}
Molecular machines are a key component in the vision of molecular nanotechnology, and have the potential to transport molecular species and cargo on surfaces. The motion of such machines should be triggered remotely, ultimately allowing a large number of molecules to be propelled by a single source, with light being an attractive stimulus. Here, we report upon the photo-induced translation of molecular machines across a surface by characterizing single molecules before and after illumination. Illumination of molecules containing a motor unit results in an enhancement in the diffusion of the molecules. The effect vanishes if an incompatible photon energy is used or if the motor unit is removed from the molecule, revealing that the enhanced motion is due to the presence of the wavelength-sensitive motor in each molecule.
\end{abstract}

Molecular machines with internal motors are fascinating objects that transform energy into useful motion at the nanoscale. ${ }^{1-6}$ In nature many processes depend on molecular motors that perform specific mechanical tasks in living cells, ${ }^{7}$ a prototypical process is the directional motion of myosin. ${ }^{8}$ Several synthetic molecular motors have been synthesized, ${ }^{2,9,10}$ including a molecular motor developed by Feringa and co-workers that rotates at a frequency in the $\mathrm{MHz}$ regime. ${ }^{11-14}$ For ultimate control of molecular machines it is essential that the motor exhibits only one sense of rotation, resulting in unidirectional translation of the molecular machine on a surface, thus only forward and no backward motion, in contrast to random motion in all directions. ${ }^{2}$ For synthetic molecular machines to contribute to the vision of nanotechnology by transporting molecular species and cargo on surfaces, the motion of 
such machines should be triggered remotely, ultimately allowing a large number of molecules to be propelled by a single source, such as light. Note that the constraints for light-driven processes are very different from those for autonomous chemically driven processes which must obey the constraints of microscopic reversibility.

While various molecular motors have been studied in solution, their investigation on surfaces is rare, due to experimental difficulties - e.g. molecule-surface interaction that inhibits molecular motion either via strong adsorption or by quenching electronic states which are required for the chemical process. However, surfaces offer important advantages: ${ }^{4}$ (i) The ability of local probe microscopy to image and characterize single molecules with submolecular resolution and to follow their motion, ${ }^{15}$ (ii) connection with the macroscopic world requires well-defined molecular locations and a solid interface, (iii) fixing the static parts of a molecular motor as for instance in the case of a single-molecule rotor ${ }^{16,17}$ and (iv) confinement on a surface, which can reduce the motor activity to two dimensions, thus reducing the complexity of the molecular process.

In an asymmetric scanning tunneling microscope (STM) junction a molecular motor adsorbed on a surface can be activated by a local stimulus, such as electron injection, or a local gradient which distorts the potential energy landscape. ${ }^{16-18}$ However, in the case of molecular machines, remote control is key for any use where not only single molecules should be addressed locally, but many molecules should be triggered in parallel. The remote control of molecular machines removes the limitation of having to address single molecules and the requirement for 'wiring' the molecules to a power source. ${ }^{19}$ Several studies have focused on laser-induced diffusion of adsorbed molecules, including the femtosecond 
irradiation of $\mathrm{CO}$ on $\mathrm{Cu}(110)^{20}$ and $\mathrm{Cu}(111),{ }^{21}$ in both cases the mechanism for diffusion is the interaction of the laser with substrate electrons.

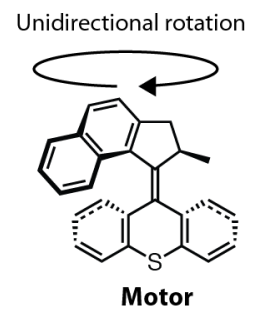

c

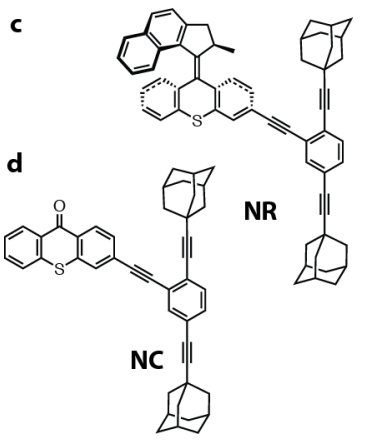

f

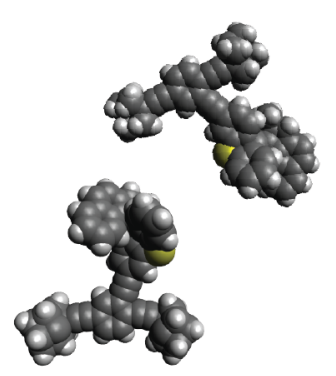

b
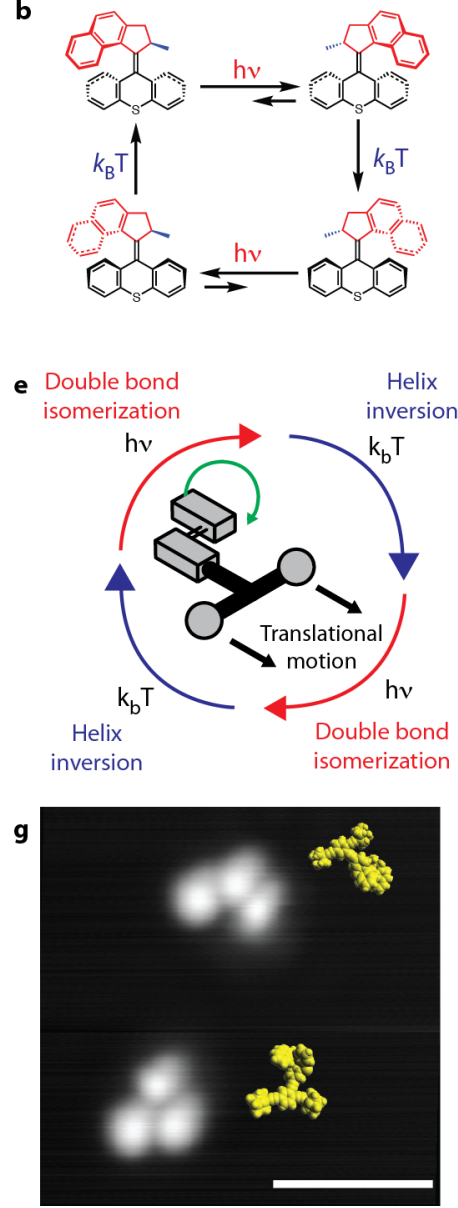

Figure 1. NanoRoadster molecule, equipped with a motor. a, Chemical structure of the molecular motor with only one sense of rotation. $\mathbf{b}$, Scheme of photo- and thermally induced steps required for operation of the motor (from ref. ${ }^{13}$ ). c-d, Chemical structures of NanoRoadster (NR) and NanoRoadster Control (NC) molecules. e, Scheme for a potential operation mode of NR via thermal and photonic excitation. f, Geometry-optimized molecular models for two possible conformations of $\mathbf{N R}$ (details in Fig. S1). g, STM image of $\mathbf{N R}$ on $\mathrm{Cu}(111)$ at $6 \mathrm{~K}\left(\mathrm{~V}_{\text {sample-bias }}=-1 \mathrm{~V}\right.$; $\mathrm{I}_{\text {tunnel }}=10 \mathrm{pA}$; scale bar $4 \mathrm{~nm}$ ); height profiles in Fig. S3. Molecular models in $\mathbf{g}$ are scaled to image dimension

For the molecular machines studied here (shown in Fig. 1), the rotation of the motor in solution (isolated structure shown in Fig. 1a) is known to be driven by (1) double bond isomerisation of the alkene 
unit connecting the stator to the rotor by photoexcitation, $\pi \rightarrow \pi^{*}$ transition, followed by (2) helix inversion via vibrational excitation as initiated by sufficient thermal energy (as discussed in reference

${ }^{13}$ ). Repetition of these two steps results in full $360^{\circ}$ rotation (Fig. 1b). So far only one motorized molecule, characterized by scanning tunneling microscopy (STM) at low temperatures, exhibited lateral motion. ${ }^{18}$ Translation has been induced locally with an STM tip, in contrast to the goal here of remote light-induced actuation. Light activation is advantageous because it can actuate many molecules at once and avoids locally deformed potential energy landscapes that occur in STM manipulation. ${ }^{22}$ Additionally, it does not require fuel molecules on the surface, which are typically used by chemically driven biological machines in solution. ${ }^{19,23}$ Such a methodology therefore offers a promising route towards remote activation, and in the present work we demonstrate that the incorporation of a wavelength-sensitive motor can be used to enhance the diffusion of functionalised molecular machines.

\section{RESULTS AND DISCUSSION}

A three-wheeled Roadster motorcycle has a single drive wheel at the rear and two wheels at the front of the vehicle, hence we termed the molecules synthesized for this study as "NanoRoadsters" (NR, Fig. 1c), one of a family of nanovehicles, termed nanocars. ${ }^{24-26}$ The use of only one motor per vehicle eliminates the need for chiral resolutions in the synthesis since each motor has only one sense of possible rotary motion. Thus, opposing directional forces that can hinder translation in the case of two or more motors, as observed previously, ${ }^{18}$ are avoided.

In analogy to operation of molecular motors in solution, the proposed concept for operation of NR (Fig. 1e) requires a combination of photoexcitation (where $h v$ is the correct energy to isomerise the 
double bond connecting the stator to the rotor) and sufficient thermal energy $\left(k_{b} T>E_{a}\right.$ for the helical inversion). To study the motion of $\mathbf{N R}$, they were deposited on an atomically flat $\mathrm{Cu}(111)$ surface under ultrahigh vacuum. Then, STM was used to follow the translation of individual molecular machines. Close-up images, acquired at $6 \mathrm{~K}$, of the internal structure of NR (Fig. 1g) allow identification of the adamantane lateral groups ('wheels') and 'motor' (further details of characterisation are given in Figure S1 and Table S1). As a brief aside, it is worthwhile to note that while the use of 'NanoRoadster', 'wheels', and 'motor' are beneficial for understanding the structure and geometry of the molecules such terms are not intended to imply an exact "nanomapping' ${ }^{27}$ of the nanoscale properties of NR to those of a macroscopic motorized vehicle.

Effect of laser illumination upon molecular diffusion. After characterizing the molecules, we assess the influence of the presence of the motor by laser illumination of the sample at $6 \mathrm{~K}$. This is done by (1) imaging individual molecules in their initial positions, (2) STM tip retraction to allow unobstructed in situ illumination of the sample and (3) tip approach to image exactly the same surface area and determine the molecular positions after illumination (see Fig. 2a, and Supporting Information (SI) for details on the difference images calculation). To get insight into the photo-sensitivity of the process, various wavelengths $\lambda(635 \mathrm{~nm}, 355 \mathrm{~nm}$ or $266 \mathrm{~nm})$ were used and compared with the same procedure without illumination. However, no diffusion of NR was observed in any of these experiments, which is in agreement with the premise that there is insufficient thermal energy for the helical inversion step at $6 \mathrm{~K}$. Hence, we conclude that the incident photons are insufficient to induce lateral translation in the absence of additional thermal energy. The photo-isomerization step probably does occur at $6 \mathrm{~K}$, as for molecular 
Switches at the same temperature, ${ }^{28}$ but a second thermal activation step is required for the motor rotation (Fig. 1b and e). One might expect the two different photo-induced isomers to result in different appearances within the STM images; however, this is not observed experimentally and is attributed to the fact that the non-planar nature of the motor unit may result in the two isomers appearing with a very similar structure in the STM data.

To investigate the effect of increased thermal energy on molecular diffusion without illumination, we varied the substrate temperature between $6 \mathrm{~K}$ and $200 \mathrm{~K}$. It is found that $\mathbf{N R}$ molecules start to diffuse at $\sim 150 \mathrm{~K}$, while for sample temperatures above $170 \mathrm{~K}$ diffusion occurs on a timescale faster than the acquisition time of the STM imaging. Therefore NR measurements with illumination were conducted within this $150 \mathrm{~K}$ to $170 \mathrm{~K}$ temperature window. Diffusion of the molecules can be determined by comparing STM images acquired before and after a specified time interval and measuring the lateral displacement of the molecules. ${ }^{15,29}$ In addition, a control molecule, NanoRoadster control (NC; Fig. 1d), which is structurally similar to NR but without a motor unit has been synthesised. The chemistry of the NC molecule is identical to that of NR, lacking only the motor unit, thereby allowing a comparison of the effect of illumination between a molecule incorporating a motor and one without. Experiments without illumination show that thermally activated lateral motion of $\mathbf{N C}$ occurs within a temperature window between $140 \mathrm{~K}$ and $160 \mathrm{~K}$, similar to that of NR. The slightly lower barrier for NC, $140 \mathrm{~K}$ compared to $150 \mathrm{~K}$ for $\mathbf{N R}$, can be attributed to the reduced interaction of $\mathrm{NC}$ with the substrate due to its smaller dimensions.

To assess the effect of the motor unit with increased thermal energy, we chose two elevated substrate temperatures (156 K and $161 \mathrm{~K}$ ) in combination with illumination. Figure $2 \mathrm{~b}$ shows STM observations 
at $161 \mathrm{~K}$ for three characteristic cases: no illumination, $\lambda=266 \mathrm{~nm}$ and $\lambda=355 \mathrm{~nm}$. The images show that several NR molecules have moved, as identified from the difference images (Fig. 2c) where the dark and bright features show the molecular position before and after illumination, respectively (see SI for additional details). In total 564 NR molecules were characterized of which 349 were observed to move (statistics are given in tables S2 and S3).
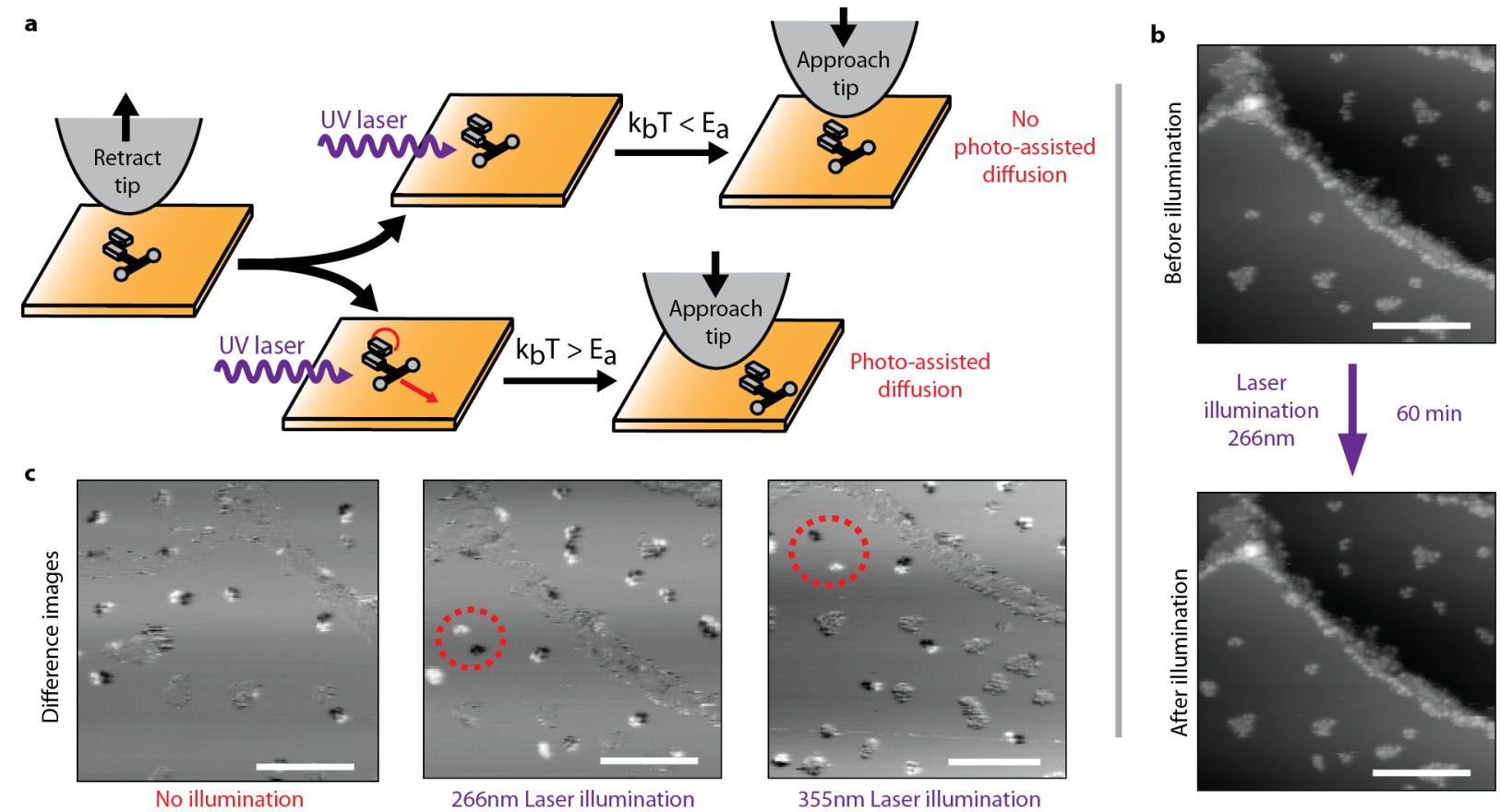

Figure 2. Illumination experiments performed on the NanoRoadster molecules. a, Scheme of experimental procedure. b, STM images of NR on $\mathrm{Cu}(111)$ acquired at $161 \mathrm{~K}$ before and after illumination with a $266 \mathrm{~nm}$ laser. c, Difference images of $\mathbf{N R}$ on $\mathrm{Cu}(111)$ acquired at $161 \mathrm{~K}$ for laser illumination experiments $(266 \mathrm{~nm}, 355 \mathrm{~nm}$, and no illumination). Difference images are obtained after drift correction by subtraction of the "before" image from the "after" image ( $\mathrm{V}_{\text {sample-bias }}=+1 \mathrm{~V}$; I $\mathrm{I}_{\text {tunnel }}$ $=10 \mathrm{pA}$; scale bars: $20 \mathrm{~nm}$ ), and are of the same area for all cases. Additional examples and complete statistics in Fig. S4 and Tables S2-S3- for NR at 161 K 564 molecules were characterized of which 349 were observed to move. 


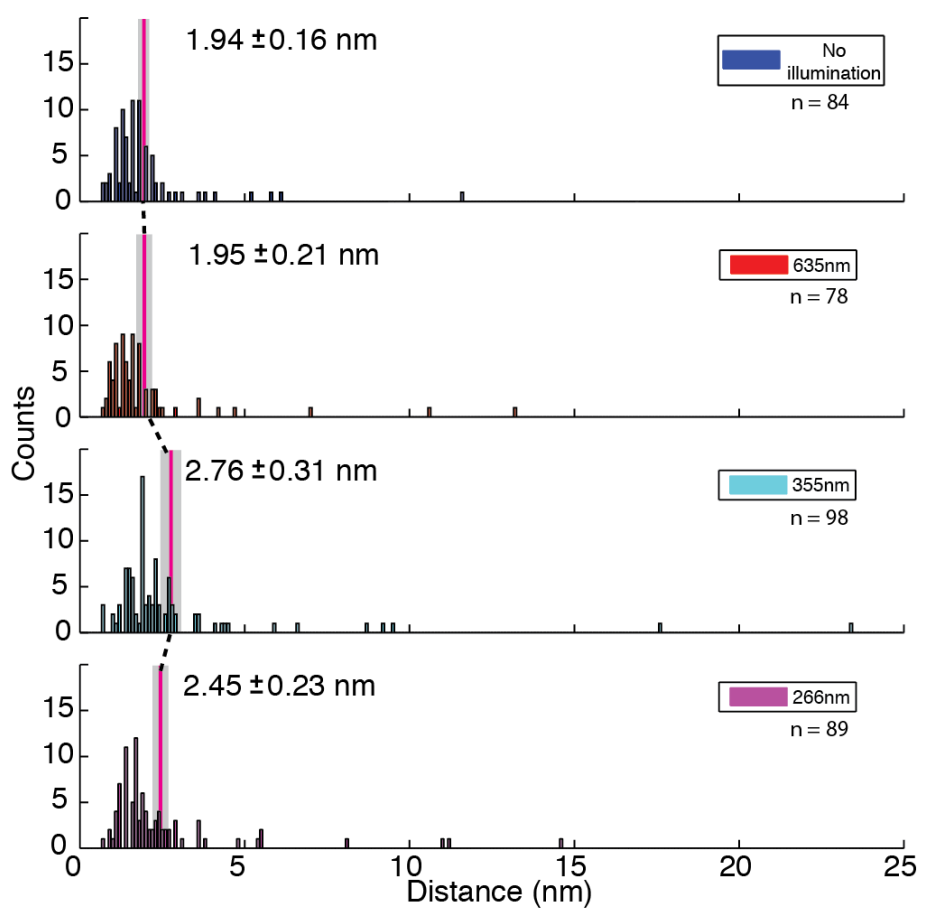

Figure 3. Histograms showing the distance moved by diffusing NR molecules after a 60 minute laser illumination for various laser wavelengths with the substrate held at $161 \mathrm{~K}$. The average distance moved (calculated from the mean value of all translation distances in the corresponding histogram) is indicated in each case by a pink bar with the error range highlighted as a grey box. The average values, and associated error bars (SE), demonstrate that the average distance moved by NR molecules illuminated with either of the UV lasers (266 nm or $355 \mathrm{~nm}$ ) is greater than that moved by molecules illuminated by the $635 \mathrm{~nm}$ laser or during the experiment without light (the total number $\mathrm{n}$ of diffusing molecules is indicated for each histogram).

A key observation is that several molecules move rather long distances of more than $5 \mathrm{~nm}$ (up to 23 $\mathrm{nm}$ per hour are observed) for the illuminated cases at $\lambda=266 \mathrm{~nm}$ and $355 \mathrm{~nm}$, but this is much less likely without illumination. At these temperatures thermal diffusion of the molecules is observed (left image Fig. 2c) and therefore a quantitative analysis to understand the enhanced diffusion induced by the laser illumination is required. The mean squared displacement (MSD) of NR is only $3.37 \pm 1.00 \mathrm{~nm}^{2}$ 
without light but $7.21 \pm 2.12 \mathrm{~nm}^{2}$ and $10.48 \pm 4.10 \mathrm{~nm}^{2}$ for illumination with $\lambda=266 \mathrm{~nm}$ and $355 \mathrm{~nm}$, respectively (full statistics are given in Tables S2 and S3). A similar trend is seen when the average distance moved by NR is considered (shown in Fig. 3), where the UV laser wavelengths (266 nm and $355 \mathrm{~nm})$ show considerably larger values $(2.45 \pm 0.23 \mathrm{~nm}$ and $2.76 \pm 0.31 \mathrm{~nm}$, respectively) as compared to the $635 \mathrm{~nm}$ laser and the case without illumination $(1.95 \pm 0.21 \mathrm{~nm}$ and $1.94 \pm 0.16 \mathrm{~nm}$, respectively) although the MSD is more appropriate to quantify distances of motion in two dimensions and can be used as the basis for determining diffusion characteristics (as shown below).

These differences in the statistical average values, well beyond the experimental error, indicate that it is indeed a light-driven process due to the presence of the molecular motor that enables the long-distance displacements, which are added to the smaller distances of the thermal motion which occur in both illuminated and non-illuminated experiments. Thus, the remotely activated motor function is likely to be responsible for molecular translation. In order to test this interpretation, we compare the NR molecule with the NanoRoadster control molecule NC (Fig. 1d), which is the same molecule but without a motor unit and should therefore exhibit substantially different behaviour.

To quantify the contribution of the motor activity to the observed molecular translation, as opposed to thermally driven diffusion, we calculate a hopping rate $h$, i.e. the number of molecules moving per second - similar to diffusion studies for non-motorized molecules, ${ }^{15,30,31}$ from:

$$
\frac{n_{\text {stat }}(t)}{N_{\text {Tot }}}=\exp (-h t)
$$

where $N_{\text {Tot }}$ is the total number of molecules observed, $n_{\text {stat }}(t)$ is the number of molecules that do not move over a time interval $t$ (Table S2). Figure 4a shows NR hopping rates for three different sample 
temperatures: $6 \mathrm{~K}, 156 \mathrm{~K}$ and $161 \mathrm{~K}$ (elevated temperatures were chosen so that diffusion occurs on a timescale accessible to STM, 800 molecules analyzed).
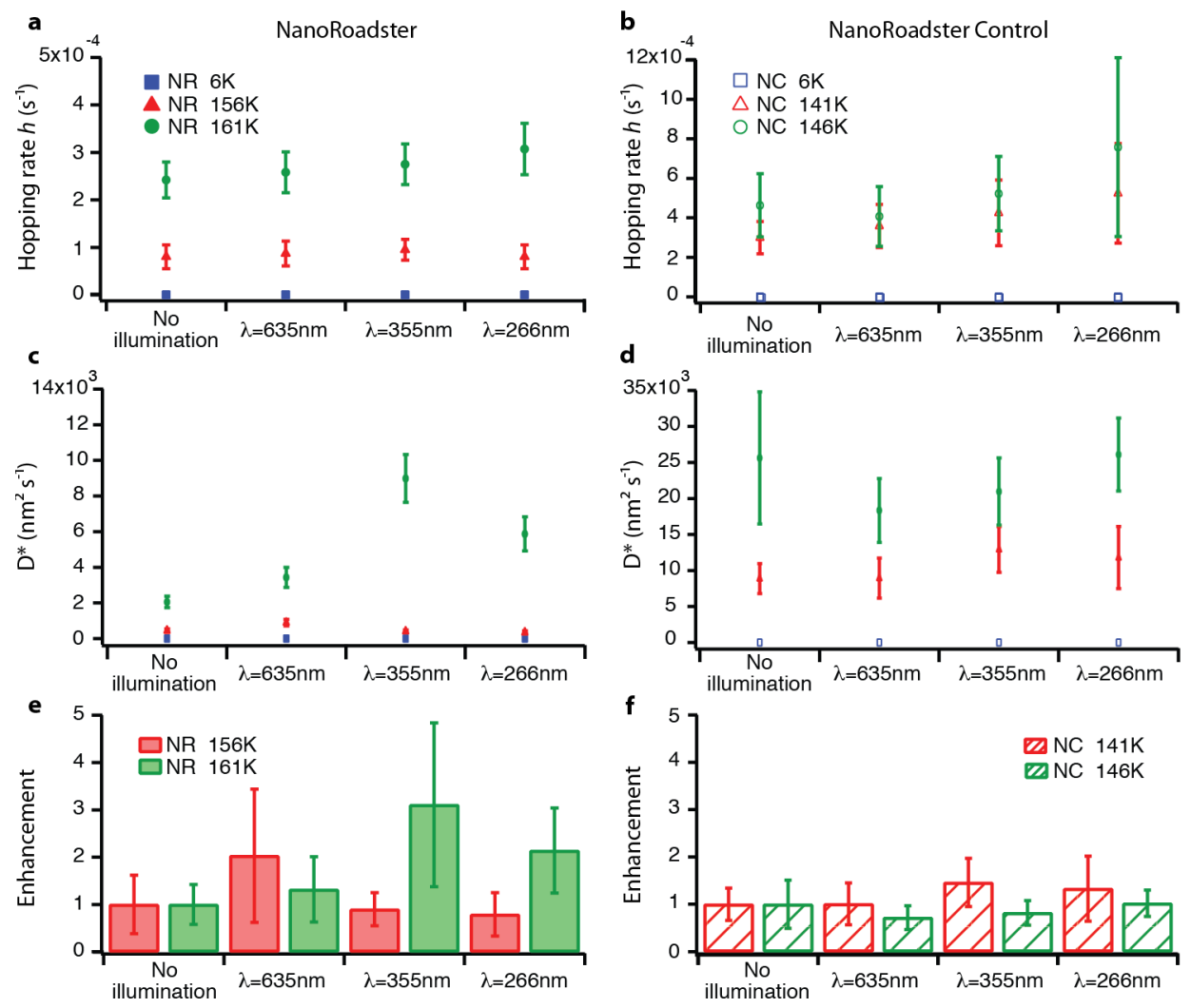

Figure 4. Statistical analysis of the molecular translation. a and $\mathbf{b}$, Hopping rate for $\mathbf{N R}$ and $\mathbf{N C}$, respectively, as obtained from Poisson statistics. $\mathbf{c}$ and $\mathbf{d}, D^{*}$ for $\mathbf{N R}$ and $\mathbf{N C}$, respectively, under different laser illumination conditions. $D^{*}$ increases for $\mathbf{N R}$ at $161 \mathrm{~K}$ when illuminated by the $355 \mathrm{~nm}$ and $266 \mathrm{~nm}$ lasers. e and $\mathbf{f}$, Enhancement of $D^{*}$ for $\mathbf{N R}$ and $\mathbf{N C}$, respectively, under different conditions. Data shown here represents the characterisation of over 1,000 molecules.

At $6 \mathrm{~K}$ no diffusion is observed and hence $h$ is zero for all cases. At $156 \mathrm{~K}$ the hopping rates are the same, within error, for the different laser illuminations. Raising the substrate temperature to $161 \mathrm{~K}$ results in an increase in $h$, irrespective of the laser wavelength, also in the experiment without light. The 
temperature-dependent results in Fig. 4a demonstrate that the number of moving NR molecules is only affected by the temperature but not by illumination, and the same is valid for NC (Fig. 4b). Hence, incident photons do not increase the likelihood of any one molecule moving and we therefore exclude that heating of the sample by the laser plays a role.

Importantly, the hopping rate $h$ concerns only the number of displaced molecules and contains no information on the distance moved by the molecules. To gain information on the translation distance, the tracer diffusion $D^{*}$ is calculated from: ${ }^{15,31,32}$

$$
D^{*}=\frac{\left\langle(\Delta r)^{2}\right\rangle}{4 t}
$$

which is valid for two-dimensional diffusion on a $\mathrm{Cu}(111)$ surface. $^{32}\left\langle(\Delta r)^{2}\right\rangle$ is the mean square displacement of the moving molecules (Table S3). Figure 4c shows $D^{*}$ for NR with different laser sources and at different temperatures (Fig. $4 \mathrm{~d}$ for $\mathrm{NC}$ in comparison). At $6 \mathrm{~K}$ no molecular diffusion is observed and $D^{*}$ is zero in all cases, while at $156 \mathrm{~K}$ finite $D^{*}$ values are measured, but almost no difference is found between the different illumination experiments. This finding implies that NR diffusion is not influenced substantially by laser illumination at $156 \mathrm{~K}$, and that the observed diffusion is predominantly thermal in origin.

The major finding is that with the substrate held at $161 \mathrm{~K}$ there is an enhancement in $D^{*}$ when the sample is illuminated with either of the two UV lasers at $355 \mathrm{~nm}$ or $266 \mathrm{~nm}$ as compared to the $635 \mathrm{~nm}$ and no-illumination experiments. This effect is highlighted in Fig. $4 \mathrm{e}$ where $D_{\text {Enhance }}^{*}\left[D_{\text {Enhance }}^{*}=\right.$ $D^{*}$ (with laser $) / D^{*}($ without laser $\left.)\right]$, the enhancement factor of $D^{*}$ caused by laser illumination, is calculated. Notably, there is a threefold enhancement in $D^{*}$ for NR molecules if they are illuminated with the $355 \mathrm{~nm}$ laser and a twofold enhancement for the $266 \mathrm{~nm}$ laser. The increase in $D^{*}$ for the 635 
$\mathrm{nm}$ laser is noticeably less pronounced. Importantly, NC lacks any such enhancement effect as the illumination of these molecules does not cause an increase of $D^{*}$, and $D_{\text {Enhance }}^{*} \sim 1$ in all cases (Fig. 4f). Note that the difference in thermal diffusion between the two species is 'normalized' by considering the enhancement factor of the tracer diffusion (Fig. 4e and 4f), and therefore the difference in thermal diffusion does not preclude the use of $\mathrm{NC}$ as a control molecule. Hence, this result shows clear evidence that individual molecular motors are remotely activated by light. Moreover, it turns out that the efficiency of the photo-sensitive motor can be tuned via the wavelength.

While these observations clearly show that the enhanced diffusion is directly related to the motor unit in the molecular structure, the exact mechanism is difficult to elucidate. In analogy to solution-phase measurements we can consider a mode of operation whereby the motor unit rotates in one direction only (as sketched in Fig.1e). The motor could also, in principle, flip back and forth between two isomers (e.g. the upper two states in Fig.1b) and therefore not complete the full cycle, at least sometimes. However, this second option seems unlikely as it does not correspond to the chemical characteristics of the motor unit, with very different rates for trans $\rightarrow$ cis and cis $\rightarrow$ trans isomerization, respectively (in particular lower rates for the latter ${ }^{33}$ ), due to the diasteriomeric transition states between the two. The proposed mechanism is therefore in concert with the well-studied unidirectional rotation of the Feringa motor, which we use. ${ }^{11-14}$

For the azimuthal orientation of the molecules on the surface a preference of the 'wheel-wheel axle' at multiples of $60^{\circ}$ relative to the $\mathrm{Cu}(111)$ surface is found (Fig. 5a), reflecting arrangement with the underlying crystal directions. It should be noted that only a rather small fraction of molecules (between about $5 \%$ and $20 \%$ ) changed their orientations (Fig. 5c) during translation. The fraction of molecules that 
change their azimuthal orientation during lateral displacement (Fig. 5c) is approximately the same small percentage for both species, NR and NC, and for all cases, without light and with light of different wavelengths, meaning that both molecules preferentially maintain their translational orientation during motion. Such behavior has been observed for non-motorized molecules on $\mathrm{Cu}(111)$ where a preferred adsorption geometry hinders changes in azimuthal orientation. ${ }^{34}$
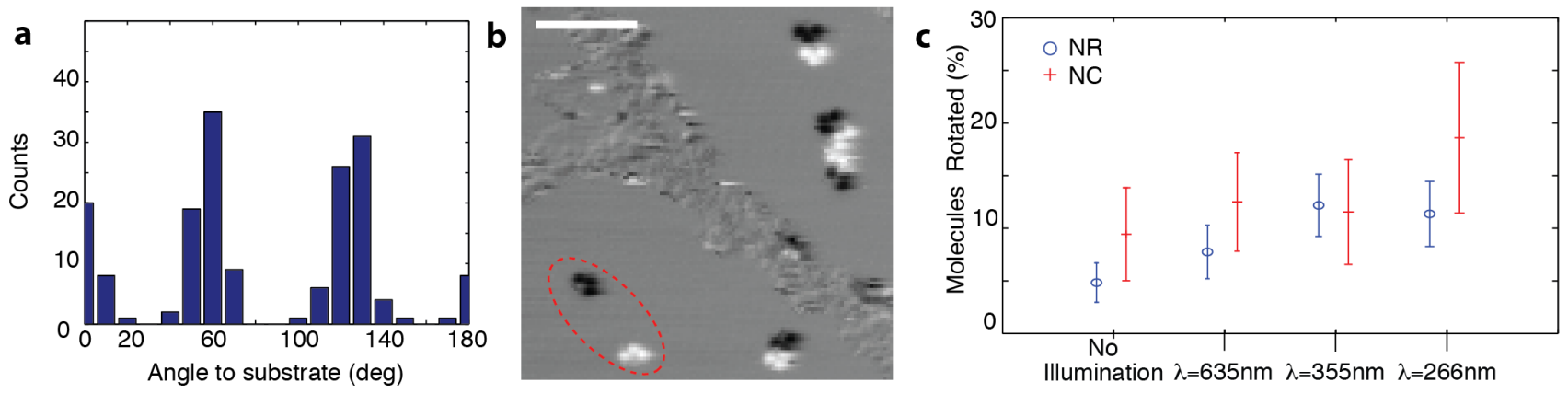

Figure 5. Azimuthal orientation of $\mathrm{NR}$ on $\mathrm{Cu}(111)$. a, Histogram showing adsorption angle made by the 'wheel-wheel axle' of NR relative to a $\mathrm{Cu}(111)$ symmetry direction. Molecules preferentially orientate at multiples of $60^{\circ}$, reflecting the substrate symmetry. b. Difference image showing translation and rotation of NR molecules ( $161 \mathrm{~K}, 355 \mathrm{~nm}$ illumination). Before and after positions of a rotated molecule are marked. Scale bar $10 \mathrm{~nm}$. c, Percentage of NR and NC molecules which rotated after laser illumination on $\mathrm{Cu}(111)$ at $161 \mathrm{~K}$ and $146 \mathrm{~K}$, respectively.

Wavelength sensitivity of the molecular motor. The pronounced wavelength sensitivity of the NR molecule to the UV photons (Fig.4e) can be directly related to the molecular motor. UV-vis absorption spectra in solution (i.e. without a surface) for the motor unit only (red spectra in Fig. 6) exhibit a strong absorbance between $340 \mathrm{~nm}$ and $400 \mathrm{~nm}$, which is attributed to a $\pi \rightarrow \pi^{*}$ transition due to the absorption of a photon by the double bond connecting the stator to the rotor in the motor unit. Importantly, this band 
is absent in the axle molecule (black spectra in Fig. 6). Hence, the $355 \mathrm{~nm}$ laser used in the surface experiments causes photoexcitation of the motor unit, in agreement with its function.

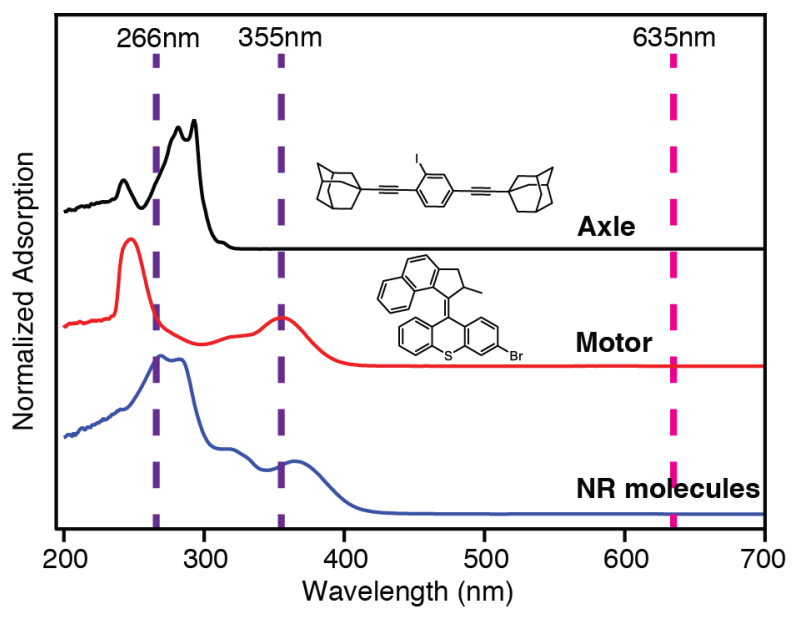

Figure 6. UV-vis spectra (in solution) and chemical structures for NanoRoadster and molecular precursors named "motor" and "axle" (as indicated). All spectra are normalized to the maximum intensity and shifted vertically for better visibility (absorption intensities are zero at wavelengths $>500$ $\mathrm{nm}$ for all spectra). The chemical structure of the NanoRoadster (NR) molecules is presented in Fig. $1 \mathrm{c}$.

While all molecular compounds absorb the UV laser wavelength of $266 \mathrm{~nm}$, it is important to note that there is no absorption band at $635 \mathrm{~nm}$ wavelength, which corresponds to the third laser used in the experiments. This is in agreement with our observation that the diffusion of molecules on the surface is strongly enhanced for the $266 \mathrm{~nm}$ and $355 \mathrm{~nm}$ wavelengths. Consequently in the case where photoisomerization of the molecular motor is coupled to diffusion, only photons with an energy suitable to induce double bond isomerization of the motor should lead to large enhancements in the molecular diffusion, which is exactly what is observed in the photo-selective increase of $D^{*}$ (Fig. 4e). Moreover, these spectra indicate that it is indeed the presence of the motor unit, and no other part, of the molecule 
which is responsible for the translation, because the molecular 'axle' and 'wheels' do not absorb above 320 nm (Fig. 6).

A small enhancement in the diffusion is also observed for $635 \mathrm{~nm}$ illumination, which does not fit to the absorption properties of the motor unit in solution (Fig.6). However, it is important that this enhancement of diffusion is also caused by the molecular motor because - equivalently to the $266 \mathrm{~nm}$ and $355 \mathrm{~nm}$ cases - it does not occur in the absence of the motor unit (Fig.4f). We tentatively assign the small $D^{*}$ increase for the $635 \mathrm{~nm}$ laser to a surface-mediated isomerization step that occurs via electronic excitation of the d-electrons of the $\mathrm{Cu}$ substrate and at smaller photon energies than those required in solution. Such a mechanism that has been reported for isomerization of an azobenzene derivative on a metal surface (with $560 \mathrm{~nm}$ illumination instead of $340 \mathrm{~nm}$ ). ${ }^{35}$

It should be noted that the surface can play an important role for functional molecules adsorbed on surfaces, in comparison with the same molecules in solution. ${ }^{36}$ However, this influence depends strongly on the precise molecule-surface interaction as for example the isomerization of azobenzene derivatives on chemically and geometrically different substrates. ${ }^{37}$ It appears from our observations that in the present case the metallic surface does not, or at least not completely, hinder the effect of the motor in $\mathbf{N R}$, in agreement with the tip-induced translational movement of a related molecule functionalized with similar motors on a supporting metallic substrate. ${ }^{18}$

The exact nature of the induced translational motion of the NR under laser illumination which lead to the observed enhancement in diffusion is not captured within our experiments, but is potentially driven by one of two mechanisms: (1) The cyclic changes in the conformation of the motor (previously termed a 'paddlewheel-like motion'26) may give rise to different preferential adsorption sites resulting in 
translational motion. (2) The different photo-induced conformations of the motor may exhibit different diffusion properties (which are not accessible under non-illuminated conditions) leading to an increase in the observed diffusion. Either of these mechanisms could give rise to the observed enhanced diffusion, and as such enhancement is not observed in the absence of the motor (NC molecule) we may attribute this effect to an inherent property of the motor.

\section{CONCLUSIONS}

Our results demonstrate that the remote operation of a motor-functionalized molecule on a surface can be used to induce translation, as evidenced by enhanced diffusion, by combining light with sufficient activation temperatures. We emphasize the importance of the critical temperature, which we propose relates to the thermal energy required for the helical inversion step of the motor rotation. If this step is not enabled, isomerization may occur but not lead to a rotation of the molecular motor and thus lateral motion on the surface is suppressed, as seen at $6 \mathrm{~K}$. However, if the temperature of the surface is too high then thermal energy dominates the process and random Brownian motion will occur. ${ }^{38}$ We find that the temperature for helical inversion, and consequently light-induced translation, of NR is similar to that required for thermal diffusion without light. This can be rationalized if one compares typical values for these processes. On the one hand, the activation barrier for helix inversion in our molecules has been determined (in solution) to be between $0.34 \mathrm{eV}$ and $0.36 \mathrm{eV} .{ }^{11}$ The molecular diffusion barrier on the other hand is for instance $0.15 \mathrm{eV}$ for benzenethiol on $\mathrm{Cu}(111)^{39}$ or between $0.41 \mathrm{eV}$ and $0.44 \mathrm{eV}$ for azobenzene on $\mathrm{Cu}(110) .{ }^{40}$ Note that benzenethiol is rather small and the diffusion barrier therefore presumably lower than in our study. Although the precise barrier height changes with the specific 
molecule-surface interaction, these values confirm that typical activation barriers for molecular diffusion on low-index copper surfaces are within about the same range as the activation energy for helix inversion. It is therefore important to accurately control both heat and light for such motor-functionalized molecules, in the same way that biological systems only function in a relatively small range of temperatures and $\mathrm{pH}$ values. ${ }^{41}$ Our results demonstrate that the remote operation of a motorfunctionalized molecule on a surface can be used to induce translation by combining light with sufficient activation temperatures. These findings open the possibility of using light to actuate large arrays of remotely powered molecular machines with concerted motions across surfaces where the demonstrated photo-sensitivity should be of great interest for further motorized molecules.

\section{EXPERIMENTAL METHODS}

STM set-up and methodology. Scanning tunneling microscopy (STM) experiments were performed under ultrahigh vacuum (UHV) conditions (base pressure $10^{-11} \mathrm{mbar}$ ), with a separate chamber (base pressure $10^{-10}$ mbar) used for the preparation of the sample. $\mathrm{A} \mathrm{Cu}(111)$ sample (MaTeck $\left.\mathrm{GmbH}\right)$ was cleaned by argon ion sputtering $\left(1.5 \mathrm{keV}, I_{\text {sample }}=\sim 4.5 \mu \mathrm{A}, P_{A r}=\sim 5 \times 10^{-6} \mathrm{mbar}, 20 \mathrm{~min}\right)$ and subsequent annealing at $\sim 500^{\circ} \mathrm{C}$ for 10 minutes. STM images were obtained over a range of temperatures $(<6 \mathrm{~K}$; liquid He cooled, and from $140 \mathrm{~K}$ to $200 \mathrm{~K}$; liquid $\mathrm{N}_{2}$ cooled with the sample counter-heated; STM data were acquired for sample temperatures of $6 \mathrm{~K}, 141 \mathrm{~K}, 146 \mathrm{~K}, 156 \mathrm{~K}$, and $161 \mathrm{~K}$ ), with an Omicron LTSTM and Nanonis Electronics. The STM was operated in constant current mode and electrochemically etched tungsten tips were used, which were covered with copper from many routine indentations into the surface for tip optimization. NR and NC were deposited from a Knudsen-cell type evaporator (Kentax 
$\mathrm{GmbH})$ at $220^{\circ} \mathrm{C}$ and $207^{\circ} \mathrm{C}$, respectively, onto a $\mathrm{Cu}(111)$ substrate held at room temperature. Note that the four adamantyl 'wheeled' variant of the NR molecule was found to be thermally labile at the required sublimation temperatures and hence could not be deposited without considerable fragmentation.

Laser illumination set-up. Laser illumination was performed in situ, with optical access to the sample for both incident and reflected beams (angle of incidence approximately $70^{\circ}$ to the surface normal) through CaF windows. In all cases laser illumination was conducted with the STM tip retracted (by several micrometres) and for a duration of 1 hour. Laser parameters: (1) $635 \mathrm{~nm}, \mathrm{CW}, 4.5 \mathrm{~mW}, \sim 0.01$ $\mathrm{cm}^{2}$ spot size. (2) $355 \mathrm{~nm}$, pulsed $-1 \mathrm{~ns}$, rep. rate $10 \mathrm{kHz}, 40.3 \mathrm{Mw}, \sim 0.06 \mathrm{~cm}^{2}$ spot size. (3) $266 \mathrm{~nm}$, pulsed $-1 \mathrm{~ns}$, rep. rate $10 \mathrm{kHz}, 7.3 \mathrm{~mW}, \sim 0.01 \mathrm{~cm}^{2}$ spot size. All lasers are s-polarized with respect to the surface.

\section{ASSOCIATED CONTENT}

Supporting Information. Supporting Information Available: Methodology for characterizing the structure of NR and NC from STM; methodology for calculating hopping rate and tracer diffusion; comparison of hopping rate, tracer diffusion, and Enhancement for NR and NC; and synthesis of NR and NC. This material is a viable free of charge via the Internet at http://pubs.acs.org.

\section{AUTHOR INFORMATION}

\section{Corresponding Author}

*Correspondence and requests for materials should be addressed to J. M. T. (tour@rice.edu) or L. G. (leonhard.grill@uni-graz.at). 


\section{Present Addresses}

\# Present address: BAM Bundesanstalt für Materialforschung und -prüfung, Berlin, Germany.

\section{Acknowledgements}

A. Saywell gratefully acknowledges financial support from a Marie Curie Intra-European Fellowship. L.

Grill thanks the German Science Foundation (DFG) for financial support via the Collaborative Research

Centre SFB 658. J. Tour acknowledges the National Science Foundation for support.

\section{REFERENCES}

(1) R. Dean Astumian. Making Molecules into Motors. Sci. Am. 2001, 57-64.

(2) Browne, W.; Feringa, B. Making Molecular Machines Work. Nat. Nanotechnol. 2006, 1, 25-35.

(3) Abendroth, J. M.; Bushuyev, O. S.; Weiss, P. S.; Barrett, C. J. Controlling Motion at the Nanoscale: Rise of the Molecular Machines. ACS Nano 2015, 9, 7746-7768.

(4) Balzani, V.; Credi, A.; Venturi, M. Molecular Machines Working on Surfaces and at Interfaces. ChemPhysChem 2008, 9, 202-220.

(5) Kay, E. R.; Leigh, D. A. Rise of the Molecular Machines. Angew. Chem. Int. Ed. 2015, 54, 1008010088.

(6) Erbas-Cakmak, S.; Leigh, D. A.; McTernan, C. T.; Nussbaumer, A. L. Artificial Molecular Machines. Chem. Rev. 2015.

(7) Heuvel, M. G. L. van den; Dekker, C. Motor Proteins at Work for Nanotechnology. Science 2007, 317, 333-336.

(8) Kodera, N.; Yamamoto, D.; Ishikawa, R.; Ando, T. Video Imaging of Walking Myosin V by HighSpeed Atomic Force Microscopy. Nature 2010, 468, 72.

(9) Morin, J. F.; Shirai, Y.; Tour, J. M. En Route to a Motorized Nanocar. Org. Lett. 2006, 8, 17131716.

(10) Kay, E. R.; Leigh, D. A.; Zerbetto, F. Synthetic Molecular Motors and Mechanical Machines. Angew. Chem.-Int. Ed. 2007, 46, 72-191.

(11) Klok, M.; Boyle, N.; Pryce, M. T.; Meetsma, A.; Browne, W. R.; Feringa, B. L. MHz Unidirectional Rotation of Molecular Rotary Motors. J. Am. Chem. Soc. 2008, 130, 10484-10485.

(12) Kulago, A. A.; Mes, E. M.; Klok, M.; Meetsma, A.; Brouwer, A. M.; Feringa, B. L. Ultrafast Light-Driven Nanomotors Based on an Acridane Stator. J. Org. Chem. 2010, 75, 666-679.

(13) Augulis, R.; Klok, M.; Feringa, B. L.; Loosdrecht, P. H. M. van. Light-Driven Rotary Molecular Motors: An Ultrafast Optical Study. Phys. Status Solidi C 2009, 6, 181-184. 
(14) Geertsema, E. M.; Molen, S. J. van der; Martens, M.; Feringa, B. L. Optimizing Rotary Processes in Synthetic Molecular Motors. Proc. Natl. Acad. Sci. 2009, 106, 16919-16924.

(15) Schunack, M.; Linderoth, T. R.; Rosei, F.; Lægsgaard, E.; Stensgaard, I.; Besenbacher, F. Long Jumps in the Surface Diffusion of Large Molecules. Phys. Rev. Lett. 2002, 88, 156102.

(16) Tierney, H. L.; Murphy, C. J.; Jewell, A. D.; Baber, A. E.; Iski, E. V.; Khodaverdian, H. Y.; McGuire, A. F.; Klebanov, N.; Sykes, E. C. H. Experimental Demonstration of a Single-Molecule Electric Motor. Nat. Nanotechnol. 2011, 6, 625-629.

(17) Perera, U. G. E.; Ample, F.; Kersell, H.; Zhang, Y.; Vives, G.; Echeverria, J.; Grisolia, M.; Rapenne, G.; Joachim, C.; Hla, S.-W. Controlled Clockwise and Anticlockwise Rotational Switching of a Molecular Motor. Nat. Nanotechnol. 2013, 8, 46-51.

(18) Kudernac, T.; Ruangsupapichat, N.; Parschau, M.; Maciá, B.; Katsonis, N.; Harutyunyan, S. R.; Ernst, K.-H.; Feringa, B. L. Electrically Driven Directional Motion of a Four-Wheeled Molecule on a Metal Surface. Nature 2011, 479, 208-211.

(19) Silvi, S.; Venturi, M.; Credi, A. Light Operated Molecular Machines. Chem. Commun. 2011, 47, 2483-2489.

(20) Bartels, L.; Wang, F.; Möller, D.; Knoesel, E.; Heinz, T. F. Real-Space Observation of Molecular Motion Induced by Femtosecond Laser Pulses. Science 2004, 305, 648-651.

(21) Zaum, C.; Meyer-auf-der-Heide, K. M.; Mehlhorn, M.; McDonough, S.; Schneider, W. F.; Morgenstern, K. Differences Between Thermal and Laser-Induced Diffusion. Phys. Rev. Lett. 2015, 114, 146104.

(22) Stroscio, J. A.; Eigler, D. M. Atomic and Molecular Manipulation with the Scanning Tunneling Microscope. Science 1991, 254, 1319-1326.

(23) Ragazzon, G.; Baroncini, M.; Silvi, S.; Venturi, M.; Credi, A. Light-Powered Autonomous and Directional Molecular Motion of a Dissipative Self-Assembling System. Nat. Nanotechnol. 2015, $10,70-75$.

(24) Chiang, P.-T.; Mielke, J.; Godoy, J.; Guerrero, J. M.; Alemany, L. B.; Villagómez, C. J.; Saywell, A.; Grill, L.; Tour, J. M. Toward a Light-Driven Motorized Nanocar: Synthesis and Initial Imaging of Single Molecules. ACS Nano 2011, 6, 592-597.

(25) Shirai, Y.; Morin, J.-F.; Sasaki, T.; Guerrero, J. M.; Tour, J. M. Recent Progress on Nanovehicles. Chem. Soc. Rev. 2006, 35, 1043-1055.

(26) Vives, G.; Tour, J. M. Synthesis of Single-Molecule Nanocars. Acc. Chem. Res. 2009, 42, 473487.

(27) Coffey, T.; Krim, J. C60 Molecular Bearings and the Phenomenon of Nanomapping. Phys. Rev. Lett. 2006, 96, 186104.

(28) Bazarnik, M.; Henzl, J.; Czajka, R.; Morgenstern, K. Light Driven Reactions of Single Physisorbed Azobenzenes. Chem. Commun. 2011, 47, 7764-7766.

(29) Sonnleitner, T.; Swart, I.; Pavlicek, N.; Poellmann, A.; Repp, J. Molecular Symmetry Governs Surface Diffusion. Phys. Rev. Lett. 2011, 107, 186103.

(30) Briner, B. G.; Doering, M.; Rust, H. P.; Bradshaw, A. M. Microscopic Molecular Diffusion Enhanced by Adsorbate Interactions. Science 1997, 278, 257-260. 
(31) Rosei, F.; Schunack, M.; Naitoh, Y.; Jiang, P.; Gourdon, A.; Laegsgaard, E.; Stensgaard, I.; Joachim, C.; Besenbacher, F. Properties of Large Organic Molecules on Metal Surfaces. Prog. Surf. Sci. 2003, 71, 95-146.

(32) Barth, J. V. Transport of Adsorbates at Metal Surfaces: From Thermal Migration to Hot Precursors. Surf. Sci. Rep. 2000, 40, 75-149.

(33) Kazaryan, A.; Kistemaker, J. C. M.; Schäfer, L. V.; Browne, W. R.; Feringa, B. L.; Filatov, M. Understanding the Dynamics Behind the Photoisomerization of a Light-Driven Fluorene Molecular Rotary Motor. J. Phys. Chem. A 2010, 114, 5058-5067.

(34) Kwon, K.-Y.; Wong, K. L.; Pawin, G.; Bartels, L.; Stolbov, S.; Rahman, T. S. Unidirectional Adsorbate Motion on a High-Symmetry Surface: "Walking'" Molecules Can Stay the Course. Phys. Rev. Lett. 2005, 95, 166101.

(35) Hagen, S.; Kate, P.; Leyssner, F.; Nandi, D.; Wolf, M.; Tegeder, P. Excitation Mechanism in the Photoisomerization of a Surface-Bound Azobenzene Derivative: Role of the Metallic Substrate. $J$. Chem. Phys. 2008, 129, 164102.

(36) Carroll, G. T.; Pollard, M. M.; Delden, R. van; Feringa, B. L. Controlled Rotary Motion of LightDriven Molecular Motors Assembled on a Gold Film. Chem. Sci. 2010, 1, 97-101.

(37) Alemani, M.; Selvanathan, S.; Ample, F.; Peters, M.; Rieder, K.; Moresco, F.; Joachim, C.; Hecht, S.; Grill, L. Adsorption and Switching Properties of Azobenzene Derivatives on Different Noble Metal Surfaces: $\mathrm{Au}(111), \mathrm{Cu}(111)$, and $\mathrm{Au}(100)$. J. Phys. Chem. C 2008, 112, 10509-10514.

(38) Astumian, R. D. Design Principles for Brownian Molecular Machines: How to Swim in Molasses and Walk in a Hurricane. Phys. Chem. Chem. Phys. 2007, 9, 5067-5083.

(39) Wong, K. L.; Kwon, K.-Y.; Bartels, L. Surface Dynamics of Benzenethiol Molecules on Cu(111). Appl. Phys. Lett. 2006, 88, 183106.

(40) Miwa, J. A.; Weigelt, S.; Gersen, H.; Besenbacher, F.; Rosei, F.; Linderoth, T. R. Azobenzene on $\mathrm{Cu}(110)$ : Adsorption Site-Dependent Diffusion. J. Am. Chem. Soc. 2006, 128, 3164-3165.

(41) Philip Nelson. Biological Physics: Energy, Information, Life; Updated First Edition.; W.H.Freeman \& Co Ltd: [s.1.], 2007.

\section{TOC graphic}

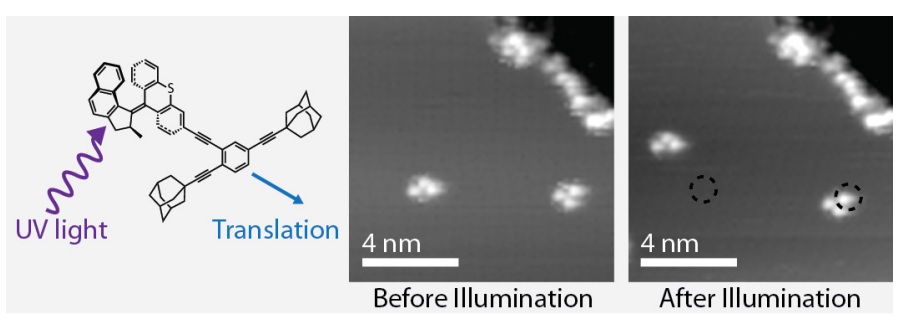

